\title{
Repulsive Magnetic Levitation-based Ocean Wave Energy Harvester with Variable Resonance: Modeling, Simulation and Experiment
}

\author{
Masoud Masoumi, Ya Wang ${ }^{1}$ \\ State University of New York, Stony Brook, NY 11790, USA
}

\begin{abstract}
This paper investigates a magnetic levitation characteristic used in a vibration based energy harvester, called repulsive magnetic scavenger (RMS). The RMS is capable of harvesting ocean wave energy with a unique repelling permanent magnet array, which provides a stronger and more uniform magnetic field, compared to its attracting magnetic counterparts. The levitating magnets are stacked together around a threaded rod so that the same pole is facing each other. Two fixed magnets placed with one at each end of the RMS provides a collocated harvesting and braking mechanism in the face of high amplitude vibrations. Magnets in the levitated magnet stack are separated by pole pieces which are made of metals to intensify the magnetic field strength. The effect of the thickness and the use of different materials with different permeability for pole pieces is also studied to obtain an optimal energy harvesting efficiency. Moreover, the procedure to find the restoring force applied to the levitating magnet stack is demonstrated. Then, the Duffing vibration equation of the harvester is solved and the frequency response function is calculated for various force amplitudes and electrical damping so as to investigate the effect of these parameters on the response of the system. Furthermore, the effect of the maximum displacement of the moving magnet stack on the natural frequency of the device is studied. And finally, Faraday's law is employed to estimate the output voltage and power of the system under the specified input excitation force. Experiments show that the output emf voltage of the manufactured prototype reaches up to $42 \mathrm{~V}$ for an excitation force with the frequency of $9 \mathrm{~Hz}$ and the maximum amplitude of $3.4 \mathrm{~g}$.
\end{abstract}

Keywords: Ocean wave energy harvester, variable resonance, repulsive magnetic levitation

\section{Introduction}

The continually crusade for environment's energy scavenging and the urge to facilitate the access to clean technologies give strong impetus to the research pertaining to the ocean wave energy harvester devices. Ocean waves are typically classified according to the state of the sea, which might appear to be very chaotic. The term wind, wave is frequently used for those waves generated by the wind, which blows and causes pressure differences on the ocean surface. The characteristics of these waves ride on the results of wind speed, the time of blowing of the wind, and the distance between the water surface and the blowing wind [1]. On the other hand, those kinds of waves, which are not being generated by the local winds, are called swell waves or surface gravity waves. The ocean encompasses both kinds of aforementioned winds, which are constantly appearing and disappearing in different directions with a seemingly topsy-turvy movement. These waves as an enormous and untamed source of clean energy, have been estimated to produce a power in the noticeable order of terawatt [2,3] and based on the estimation of The International Energy Agency, wave energy has the potential to provide over $10 \%$ of the world's current electricity demand. Also, based on the world wave energy resource map, which represents the estimation of average energy flux of the ocean waves for the whole globe, energy flux of the waves on the west coast and east coast of the US can be estimated circa 40 and $20 \mathrm{~kW} / \mathrm{m}$ respectively [4].

\footnotetext{
${ }^{1}$ Corresponding Author: Ya Wang, Ph.D. Assistant Professor, Department of Mechanical Engineering, State University of New York, Stony Brook, Email: ya.s.wang@ stonybrook.edu, Phone: 631-632-8322, Fax: 631-632-8355.
} 
Since the first patented device for harvesting ocean wave energy [5], countless efforts have been made to propose a high-efficient, low-cost, and feasible method to harness the waves' energy. These devices can be categorized according to the mechanisms, which are employed to convert the kinetic energy of the waves into the electrical energy. Number of wave energy converters which were patented in North America, Japan and Europe until 2002 reaches to over 1000 [6] and all of these systems can be classified into four major mechanisms [6], including an oscillating water column, mechanisms which use the energy of indecent waves to drive low head turbines, floating or sea-bed-mounted point absorbers which utilize a mechanical/hydraulic system to drive an electrical generator, and finally those devices that drive a deflector or a flexible bag by capturing the horizontal wave motions.

The number of projects and researches conducted by different universities and research institutes in different countries is eye-catching. First oscillating water column device was used to navigate travelling ships, working as an audible warning device in the coast of the USA in the nineteenth century. Japan was the first country which built and installed a real-size wave energy converter to drive a turbine for electricity production with the power output of 70-500 W for each unit [7]. In 1974, Stephen Salter invented his Nodding Duck wave converter which was called "Edinburgh Duck" and performed some experimental tests on the small-scale device. Also in the 1970s, Falnes and Kjell were conducting experimental tests and numerical analysis on surface wave energy harvesting methods [8, 9]. First researches in the US were conducted by Michael E. McCormick [9].

K. Rhinefrank et all [10], designed, built and studied a bouy-type ocean wave energy harvester, where a permanent magnet linear generator was used to harvest the vertical components of ocean waves. Simulation results and experimental outcomes were both reported. K. Thorburn and M. Leijon [11] conducted a study on the procedure of connecting ocean wave energy harvesters together to create a farm and performed different simulations so as to find the power output for a farm with 5-10 units. M.J. Brennan et all [12] theoretically studied the Duffing oscillation with a linear viscous damping to provide a full set of equations using the harmonic balance method. They compared their results with the ones from direct integration approach and also the perturbation method. N. M. Kimoulakis et all [13] put forward a methodology to design and model a four-sided linear power generator as an ocean wave energy harvester with a moving part of a series of magnets inside a stator winding. They used the velocity of $0.9 \mathrm{~m} / \mathrm{s}$ for the floating buoy and tried different types of magnetic configurations in terms of their positions against the stator.

D. Elwood et all [14] reported a modelling approach and also experimental results for a similar system. Their device, SeaBeaveI, consists of an outer layer of magnets vertically moving against a central taut-moored which is holding the armature of the linear generator. Similarly, J. Prudell et all [15] performed a FE-modelling and experiments on a tubular permanent magnet linear generator as a wave energy scavenger. Their device is slightly similar to what was reported by D. Elwoon et all [14], however, they specifically determined the dimensions of the air-gaps and magnets to target the desired frequencies. Despite all the conducted studies, the specific magnet arrangement and structure which are discussed in this paper has not been a subject of a study to the best of our knowledge. Also, they did not use permanent magnets at both ends to provide a braking system and to add non-linearity properties to the harvester.

This paper presents a parametric, numerical and experimental study of a repulsive magnetic scavenger (RMS) with a specific arrangement of stacked magnets, which produces a nonlinear vibration levitation using repelling magnetic forces. The objective of this study is to lay the foundation to develop a high power density broadband ocean wave energy harvester with variable resonance. After explaining the structure of the device at the first section, a parametric and numerical study is carried on for each substructure of the harvester and the output voltage (power) under the sinusoidal excitation force is predicted. In the end, a prototype is fabricated and experimental verification is performed.

\section{Structure of the device}

The schematic diagram of the RMS is shown in Fig. 1. Its levitation mechanism provides us with a very useful advantage over the traditional permanent magnet generators in terms of magnet arrangement 
and magnetic braking system, which results in a much stronger magnetic flux density. In particular, the repelling magnet stack generates a stronger magnetic field than that of an attracting magnetic stack, and the end magnets recycle the potential energy often lost in conventional spring brakes. When the RMS is under vibrational environment, the middle levitating magnets stack oscillates in a nonlinear way of approaching two end magnets. The nonlinear nature of the magnetic levitation allows the resonance of the RMS to be tuned by simply changing the spacing between end magnets.

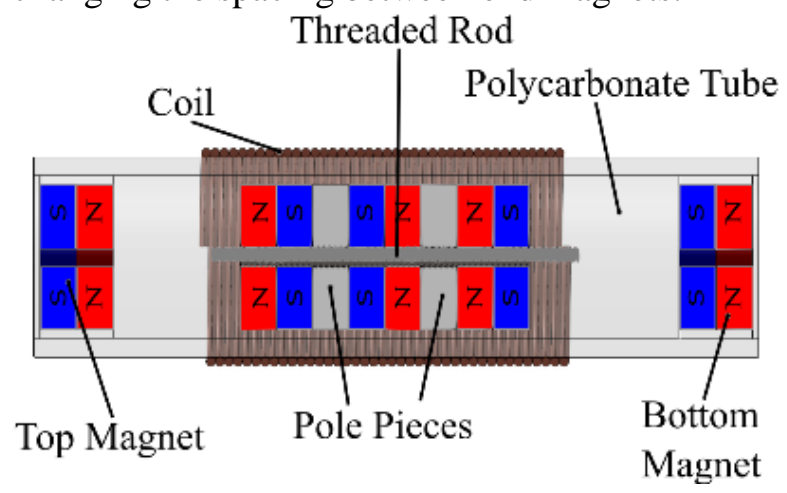

Fig. 1 Structure of the device

\section{Parametric Study and Numerical Simulation}

Here, the magnetic field generated by various magnet arrangements of the RMS is simulated using a finite element (FE) software in different steps. First, the effect of different magnet arrangements and the resulted magnetic field strength are studied. After deriving the governing equations of the system, the most efficient configuration is chosen to further study its restoring force. Finally, such a configuration is used to simulate the vibration response both in the time domain and the frequency domain in order to further estimate its voltage and power output. Magnetic field simulations have been conducted by FEMM 4.2 software and also MATLAB ${ }^{\mathrm{TM}}$.

\subsection{Pole directions}

In order to further characterize and then control the distribution of the resulting magnetic field, the magnetic flux density of two different configurations is studied for two magnets: same poles facing each other and opposite poles facing each other. Their corresponding magnetic properties are listed in table 1.

Table 1 Magnet properties

\begin{tabular}{cc}
\hline Property & Value \\
\hline Permanent magnet outer diameter & 2 in. $(50.8 \mathrm{~mm})$ \\
Permanent magnet inner diameter & $0.25 \mathrm{in}(6.35 \mathrm{~mm})$ \\
Thickness of each magnet & 1 in $(15.4 \mathrm{~mm})$ \\
Magnetization & $1.03 \times 10^{6} \mathrm{~A} / \mathrm{m}$ \\
Permanent magnet composition & NdFeB grade 42 \\
\hline
\end{tabular}

In order to have a good vision about the magnetic field distribution passing through the coil around the RMS, the normal and the tangential magnetic field strength is simulated, passing through the space at 0.2 inches $(5.08 \mathrm{~mm}$ ) away from the magnet stack in the transverse direction (see Fig. 2). This location $\left(d_{1}\right)$ is supposed to be the location of the coil and therefore the quantification of the magnetic field passing through the line $d$, is critical for predicting the power output of the system. The results of the tangent and normal flux density for these configurations along the line $d$ are shown in Fig. 2. 


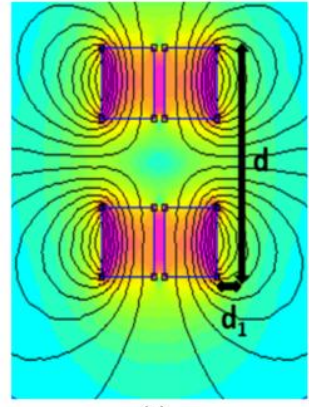

(a)

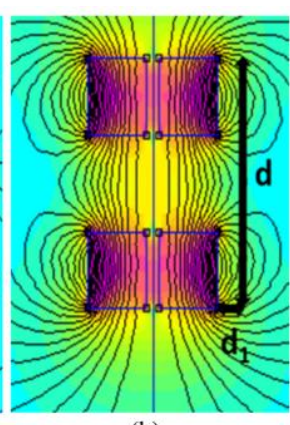

(b)

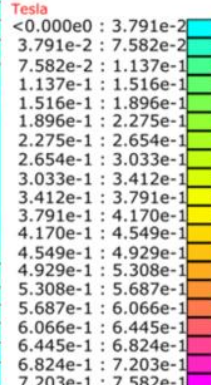

(c)

Fig. 2 (a) same poles facing each other, (b) opposite poles facing each other $(\mathrm{d}=3.25 \mathrm{in}$. $(82.55 \mathrm{~mm}) \&$ $\mathrm{d}_{1}=0.2$ in. $\left.(5.08 \mathrm{~mm})\right)$, (c) magnetic field scale

As shown in Fig. 3 (a) and (c), the maximum normal flux density happens to be around 0.3 Tesla compared to 0.22 Tesla for the other scenario. In this figure, $d$ is the same parameter shown in Fig.2 (a) and (b). Going from $d=0$ to $d=3.25 \mathrm{~mm}$ in Fig. 3 is equivalent to moving from one side of the vertical arrow to the other side in Fig. 2 and simultaneously measuring the normal and tangential magnetic flux densities. Also, the magnetic field varies around 0.1 Tesla between the two magnets and it maintains a high value compared to the other arrangement in which the magnetic field varies more noticeably between the two magnets and drops to zero in the middle. Having a stronger magnetic field at a distance between the two magnets leads to a higher output current for the same coil with the same moving speed.

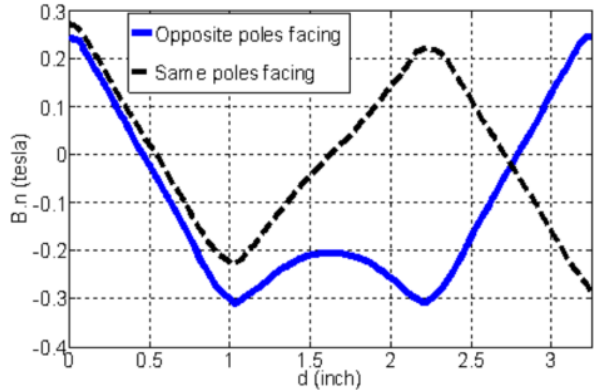

(a)

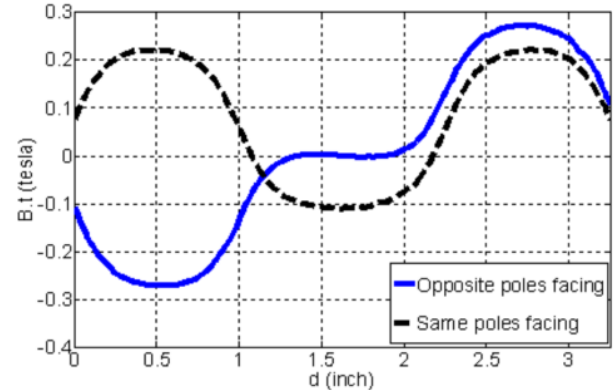

(b)

Fig. 3 (a) Normal flux density (b) Tangential flux density

\subsection{Pole piece material}

The pole piece refers to the metal used to fill the gap between two magnets. Since it is nearly impossible to attach two magnets together with the same pole facing each other, we need consider a distance between these magnets and this distance can be filled with a metal or left empty (air gap). Here, we consider different gap fillers for pole pieces and compare their performance in a numerical way to determine which one would better serve our objectives. The relative permeabilities of the common filling materials to conduct this study are provided in Table 2 .

Table 2 Relative permeability for pole pieces

\begin{tabular}{ccccccc}
\hline Material & \multirow{2}{*}{ Air } & $\begin{array}{c}\text { Carbon } \\
\text { Steel }\end{array}$ & $\begin{array}{c}\text { Iron } \\
99.8 \%\end{array}$ & $\begin{array}{c}\text { Cobalt } \\
\text { Iron }\end{array}$ & \multirow{2}{*}{ Nanoperm } & Iron \\
Permeability & 1 & 100 & 5000 & 18,000 & 80,000 & 200,000 \\
\hline
\end{tabular}

Figure 4 shows a comparison between the normal flux density for different gap fillers, ranging from a carbon steel with a very low relative permeability (around 100) to a 99.95\% Iron with a high relative 
permeability (around 200000). This comparison shows that a metal filling increases the magnetic field intensity than that of air filling. However, there is no noticeable difference between metals with different permeabilities in terms of improving its magnetic field intensity. Therefore, a more cost effective metal (1018 steel) is chosen in this work as pole pieces.

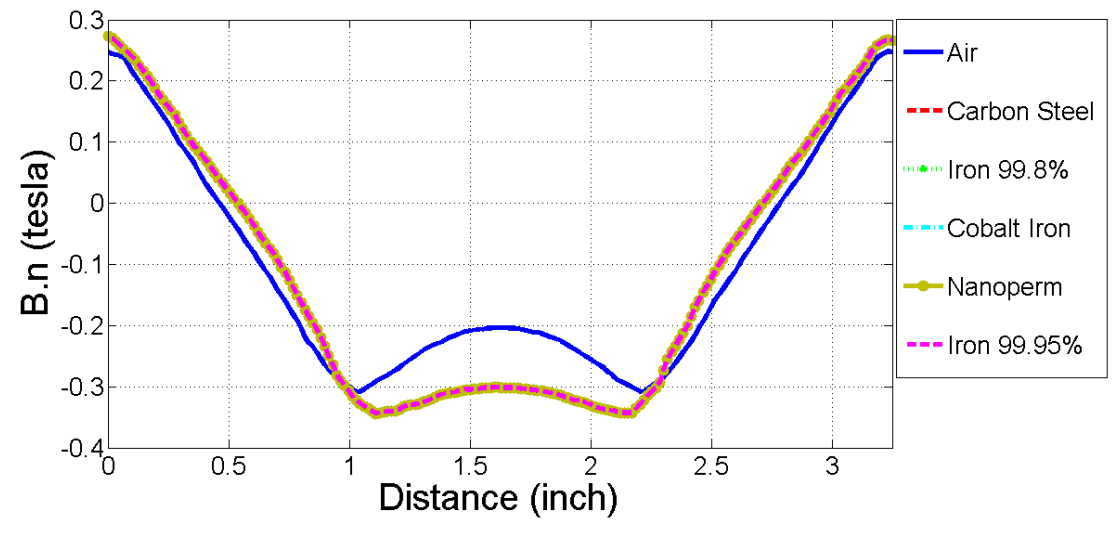

Fig. 4 Normal flux density of different materials as the pole piece

\subsection{Pole piece thickness}

The effect of the pole piece thickness on the strength and the distribution of the resulting magnetic field intensity is simulated in this section. A 1018 carbon steel is used as pole piece material in these simulations.

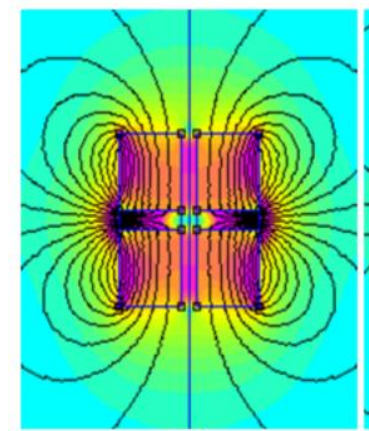

(a)

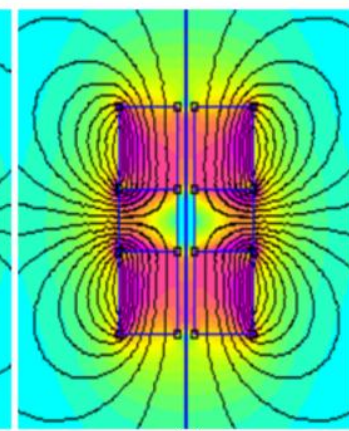

(b)

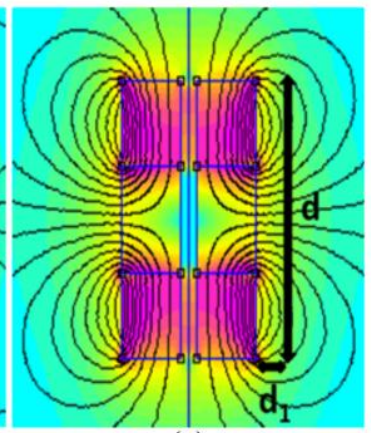

(c)

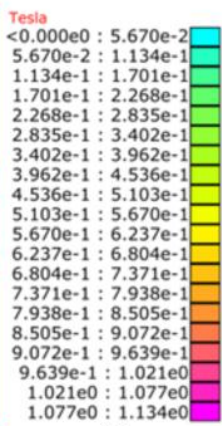

(d)

Fig. 5 Magnetic field simulation for different magnet distance: (a) $d=2.25$ in. $(57.15 \mathrm{~mm}) \& d_{1}=0.2$ in. $(5.08 \mathrm{~mm}),(b) \mathrm{d}=2.75$ in. $(69.85 \mathrm{~mm}) \& \mathrm{~d}_{1}=0.2$ in. $(5.08 \mathrm{~mm}),(\mathrm{c}) \mathrm{d}=3.25 \mathrm{in} .(82.55 \mathrm{~mm}) \& \mathrm{~d}_{1}=0.2$ in. $(5.08 \mathrm{~mm}),(\mathrm{d})$ magnetic field scale
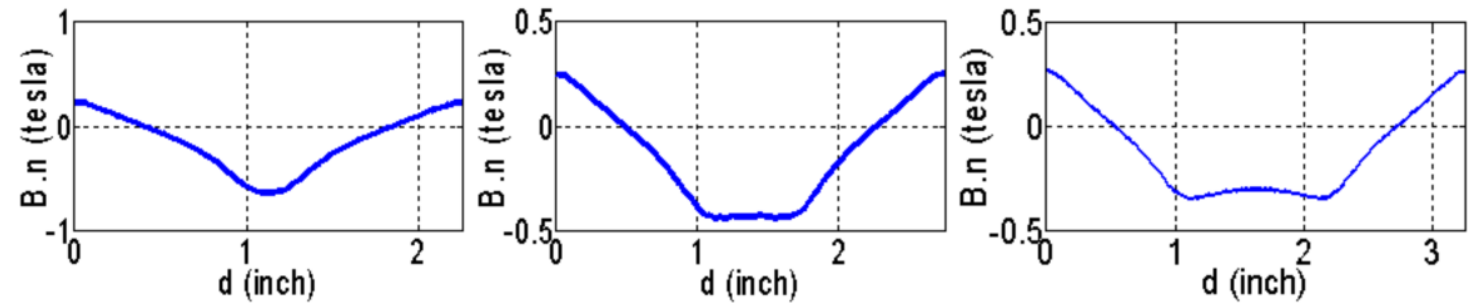

Fig. 6 Normal magnetic flux density along the line $d$ : (a) $d=2.25$ in. \& $d_{1}=0.2$ in (b) $d=2.75$ in. \& $d_{1}=0.2$ in (c) $\mathrm{d}=3.25$ in. $\& \mathrm{~d}_{1}=0.2$ 
The normal flux density of each case along the lined is shown in Fig. 6. As expected, the normal magnetic field intensity becomes stronger by reducing the pole piece thickness. However, the thinner the pole piece the huge amount of force required to assemble the magnets together. In our case, the pole piece of 0.5 in $(12.7 \mathrm{~mm})$ thick is used and therefore $d$ would be 2.5 in $(63.5 \mathrm{~mm})$.

\subsection{Governing Equation}

The magnetic levitation stack can be modelled as a single-degree-of-freedom base-vibration system and its nonlinear Duffing equation is in the form of [16]:

$m \ddot{x}+c \dot{x}+k x+\alpha x^{3}=F \cos (\omega t+\varphi)$

where, $x$ is the relative displacement, $m$ is the effective mass of the moving magnet stack, $F \cos (\omega t+\varphi)$ is the harmonic excitation force and finally $c$ is the effective damping coefficient, which can be found from $c=c_{m}+c_{e}$. The coefficient $c_{m}$ is the mechanical damping and $c_{e}$ is the electrical damping as follows

$c_{e}=\frac{\phi^{2}}{\left(R_{i}+R_{o}\right)}$

where, $\phi$ is the electromechanical coupling coefficient, $R_{i}$ is the internal resistance of the coil and $R_{o}$ is the external load resistance. The electromechanical coefficient can be found from $\phi=N B L$, where, $N$ is the number of coil turns, $B$ is the average strength of the magnetic field and $L$ is the length of the coil. The system stiffness in the Eq. (1) (i.e., $x+\alpha x^{3}$ ) is determined by the restoring force generated by the repelling force between the levitating magnet stack and the two end magnets. In order to identify the coefficients $k$ and $\alpha$, the repelling magnetic force can be measured and then curve fitted following the steps listed in [17]: first, fit a curve to the force measured from the magnet stack in the form of $F_{s}(s)=\sum_{n=0}^{3} a_{n} s^{n}$

For the top and bottom magnets, we substitute $s$ with $\left(d_{0}-x\right)$ and $\left(d_{0}+x\right)$ respectively:

$F_{t}(x)=\sum_{n=0}^{3} a_{n}\left(d_{0}-x\right)^{n}, \quad F_{b}(x)=\sum_{n=0}^{3} a_{n}\left(d_{0}+x\right)^{n}$

The total restoring force is given by a vector summation of the restoring forces acting on the top and bottom magnets

$F(x)=F_{b}(x)-F_{t}(x)=\left(2 a_{1}+4 d_{0} a_{2}+6 d_{0}^{2} a_{3}\right) x+2 a_{3} x^{3}$ which means $k=2 a_{1}+4 d_{0} a_{2}+6 d_{0}^{2} a_{3}$ and $\alpha=2 a_{3}$.

\subsection{Vibration Response}

In this section, the vibration response is simulated and then the emf voltage and power output of the system is predicted.

\subsubsection{Restoring force coefficient identification}

A numerical simulation is performed to figure out the restoring force between the magnet stack and top magnet, and its magnetic field intensity is shown in Fig. 7. 

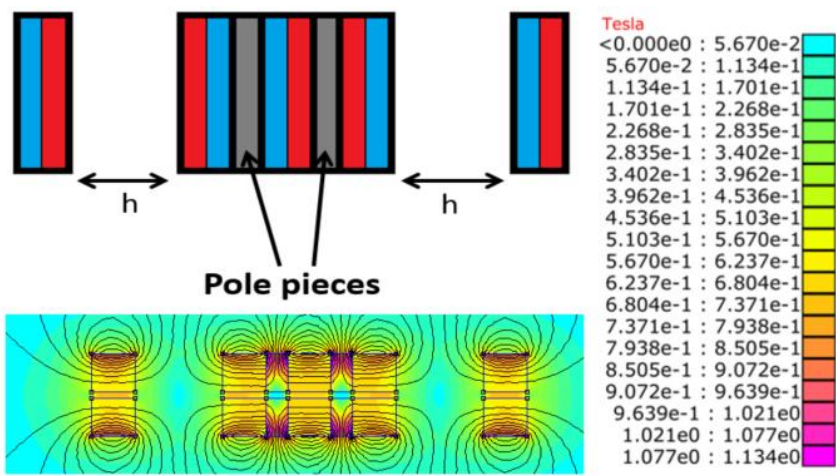

Figure 7 structure of the device, including magnets and the pole pieces (see Table 3 for dimensions), magnetic field simulation of the device when the moving magnet stack is in the center and the magnetic field scale

Table 3 Magnets properties for the RMS

\begin{tabular}{cc}
\hline Property & Value \\
Permanent magnet outer diameter & 2 in. $(50.8 \mathrm{~mm})$ \\
Permanent magnet inner diameter & 0.25 in. $(6.35 \mathrm{~mm})$ \\
Magnetization & $1.03 \mathrm{e} 6 \mathrm{~A} / \mathrm{m}$ \\
Permanent magnet composition & NdFeB grade 42 \\
$h$ & 2 in. $(50.8 \mathrm{~mm})$ \\
Pole piece (1018 Steel) thickness & 0.5 in. $(12.7 \mathrm{~mm})$ \\
Magnet thickness & 1 in. $(25.4 \mathrm{~mm})$ \\
\hline
\end{tabular}

So as to identify the restoring force due to the repelling between the magnets, the restoring force versus distance between magnet stack and the top magnet is curve fitted against the values simulated using a finite element software FEMM 4.2, shown in Fig. 8. The x-axis label, "distance" mentioned in this figure is presenting the distance between two magnets. It starts from very close distance between two magnets and goes up to $0.06 \mathrm{~m}$ where the last evaluation was made.

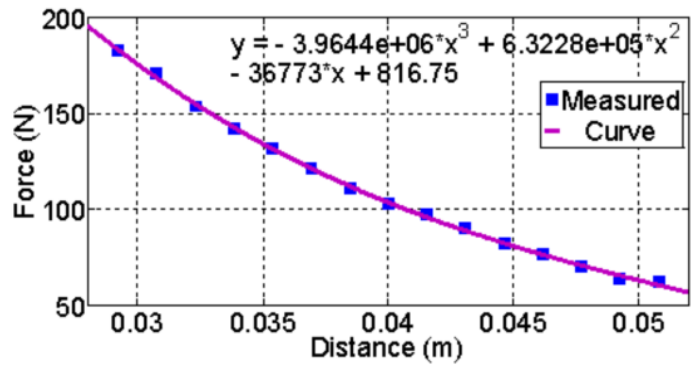

Fig. 8 Restoring force versus distance for the top magnet distance from the end-fixed magnet

A curve is fitted to the simulated force and Eqs.(4-6) can be used to find the coefficients $k$ and $\alpha$ in Eq.(1) as follows:

$F_{t}(x)=\sum_{n=0}^{3} a_{n}\left(d_{0}-x\right)^{n}=816.75-36773\left(d_{0}-x\right)+632280\left(d_{0}-x\right)^{2}-3.9644 \times 10^{6}\left(d_{0}-x\right)^{3}$ 
$F_{b}(x)=\sum_{n=0}^{3} a_{n}\left(d_{0}+x\right)^{n}=816.75-36773\left(d_{0}+x\right)+632280\left(d_{0}+x\right)^{2}-3.9644 \times 10^{6}\left(d_{0}+x\right)^{3}$

And finally,

$$
F(x)=F_{b}(x)-F_{t}(x)=-6450.84 x-7.9288 \times 10^{6} x^{3}
$$

\subsubsection{Frequency response calculations}

In order to solve Eq. (1), we first non-dimensionalize it as a conventional procedure [16]:

$u^{\prime \prime}+2 \zeta u^{\prime}+u+\beta u^{3}=\cos (\Omega \tau+\varphi)$

In which, we have $u=k x / F, \omega_{n}=\sqrt{k / m}, \Omega=\omega / \omega_{n}, \beta=\alpha F^{2} / k^{3}, \zeta=c / 2 m \omega_{n}$, and $\tau=\omega_{n} t$.

By substituting $=U \cos (\omega t)$, we get

$\frac{9}{16} \beta^{2} U^{6}+\frac{3}{2} \beta\left(1-\Omega^{2}\right) U^{4}+\left[\left(1-\Omega^{2}\right)^{2}+(2 \zeta \Omega)^{2}\right] U^{2}=1$

To have some insights into the nonlinear phenomenon, we find the nonlinear frequency response $(U)$ for the values listed in Table 4 (see Fig. 9). It should be noted that only $\alpha$ and $k$ and $m$ are the parameters obtained from our device and the rest of the parameters are used based on [17].
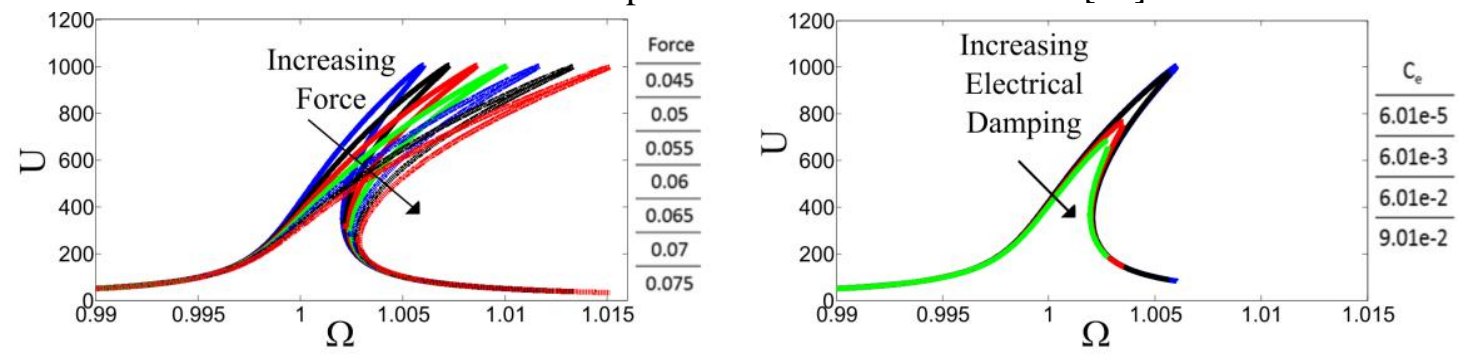

Fig. 9 Frequency responses of the device under the assumption of using the values in Table 4 for: (a) nondimensional force values listed in the figure, (b) non-dimensional electrical damping values listed in the

figure

Table 4 Vibration parameters used to simulate the dynamics of the system

\begin{tabular}{cc}
\hline Property & Value \\
\hline$\alpha\left(\mathrm{N} / \mathrm{m}^{3}\right)$ & $2.778 \times 10^{7}$ \\
$K(\mathrm{~N} / \mathrm{m})$ & $1.634 \times 10^{4}$ \\
$m(\mathrm{Kg})$ & 2.268 \\
$c_{e}(\mathrm{~N} \mathrm{~s} / \mathrm{m})$ & $6.010 \times 10^{-5}$ \\
$c_{m}(\mathrm{~N} \mathrm{~s} / \mathrm{m})$ & 0.190 \\
\hline
\end{tabular}

\subsubsection{The voltage/power output under a sinusoidal base excitation}

Here, the vibration response of the levitation stack of the RMS in time domain is simulated by using the parameters pertaining to the designed system. The Runge-Kutta numerical method is used to numerically solve Eq. (1) and then the results (i.e., velocity and displacement) were used as inputs to the FEMM 4.2 software to translate the moving magnet stack. Finally, the obtained magnetic field from each step is used together with the velocity and the displacement of the stack to calculate the output voltage by using the Faraday's law:

$e m f=-N \frac{d \phi}{d t}=-N \frac{d \phi}{d y} \frac{d y}{d t}$ 
where, $N$ is the number of coil turns. In this study, ten layers of an AWG 36 wire are wound around the RMS for the height of 4 inches.

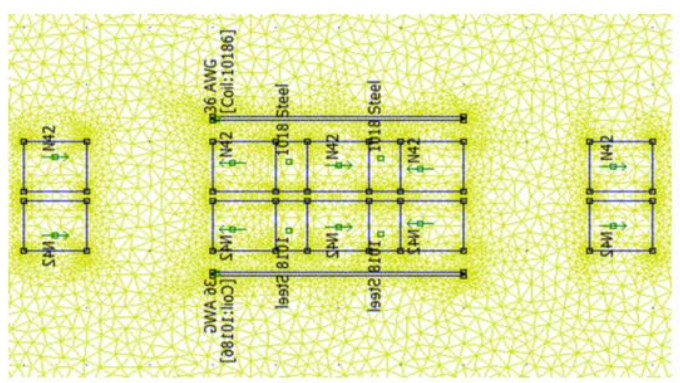

Fig. 10 FE model of the RMS

The number of turns will be $N=10186$ and the resistance of the coil is $R_{i}=6190.58 \mathrm{Ohm}$. The Finite Element Model of the RMS is shown in Fig. 10 and the values used to find the time-response are listed in Table 5.

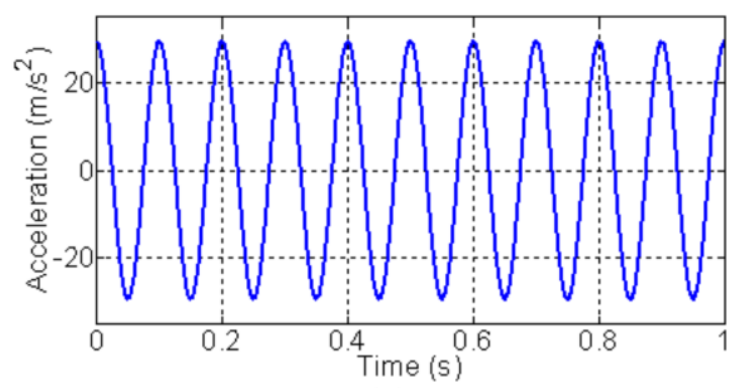

(a)

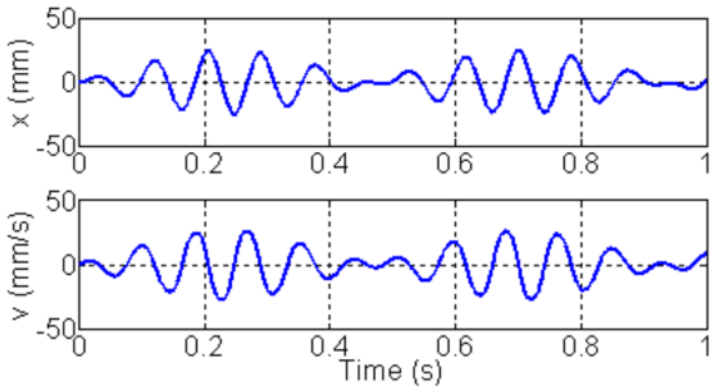

(b)

Fig. 11 (a) Excitation force (b) Velocity and Displacement of the device under the vibrations with the characteristics listed in Table 5

Table 5 Characteristics of the device system (see Table 3 for magnets arrangement and properties)

\begin{tabular}{ccccccccc}
\hline $\begin{array}{c}\text { Mass of } \\
\text { the stack } \\
(\mathrm{Kg})\end{array}$ & $\begin{array}{c}\alpha \\
\left(\mathrm{N} / \mathrm{m}^{3}\right)\end{array}$ & $\begin{array}{c}k \\
(\mathrm{~N} / \mathrm{m})\end{array}$ & $\begin{array}{c}\text { Height of } \\
\text { the coil }(\mathrm{in})\end{array}$ & $\begin{array}{c}\text { Coil } \\
\text { turns }\end{array}$ & $\begin{array}{c}\text { Internal } \\
\text { resistance } \\
(\mathrm{Ohm})\end{array}$ & $\begin{array}{c}\text { Natural } \\
\text { Frequency } \\
(\mathrm{Hz})\end{array}$ & $\begin{array}{c}\text { Excitation } \\
\text { frequency } \\
(\mathrm{Hz})\end{array}$ & $\begin{array}{c}\text { Amplitude of } \\
\text { excitation } \\
\text { force }\end{array}$ \\
1.539 & $7.92 \times 10^{6}$ & 6450.84 & $4(101.6 \mathrm{~mm})$ & 10186 & 6190.58 & 10.3 & 10 & $3 \mathrm{~g}$ \\
\hline
\end{tabular}

Using the displacement and velocity provided in Fig. 11(b), which are found from Eq.(7), and implementing Eq. (9), voltage output can be found as shown in Fig. 12. 


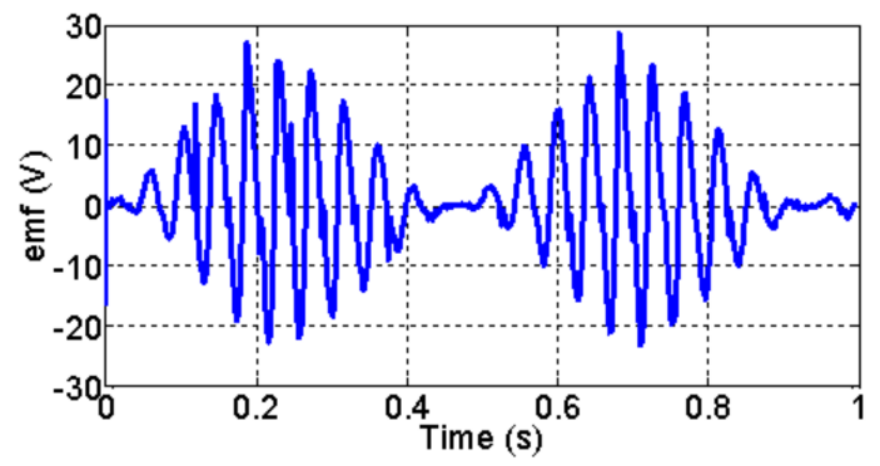

Fig. 12 Voltage output of the device under the vibration with the characteristics listed in Table 5

Also, the power output considering an external load resistance of $1 \mathrm{k} \Omega$ along with the internal load resistance due to the coil is calculated and shown in Fig. 13.

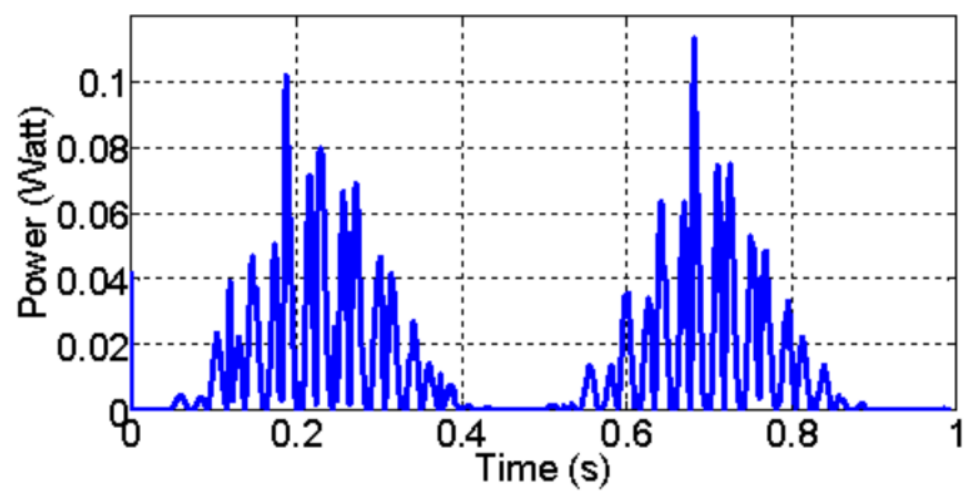

Fig. 13 Power output of the device under the vibration with characteristics listed in Table 5

\subsection{On the natural frequency and the RMS design}

When it comes to design a device for energy harvesting, the main focus is to manufacture a system which has the closest frequency to the frequency of the vibration source. One of the specifications of this nonlinear system is its variable resonance frequency based on the amplitude of the vibration of the magnet stack. Due to the nonlinear nature of the magnet stack levitation, the system can be modelled as a hardening system, in which its stiffness increases by increasing the magnetic force. As it can be seen from Fig. 14, by increasing the force applied to the magnet stack and as a result, increasing the maximum displacement of the magnet stack during the vibration, the natural frequency of the system changes. Due to the fact that the mass of the system is constant for each case, changing the maximum displacement leads to changing the value of the restoring force and therefore altering the stiffness of the system (see Eq.(6) and the line following).

Figure 14 shows the pattern of changing the natural frequency of the current design versus maximum displacement of the magnet stack (please note that the distance between the magnet stack and top or bottom magnet is 2 inches at the beginning and before the system starts oscillating). As shown in Fig. 14, the natural frequency decreases with the increase of the magnet stack maximum displacement. However, it has a minimum value in which the system starts showing the hardening properties, meaning the stiffness of the system increases and as a result, the natural frequency increases. 


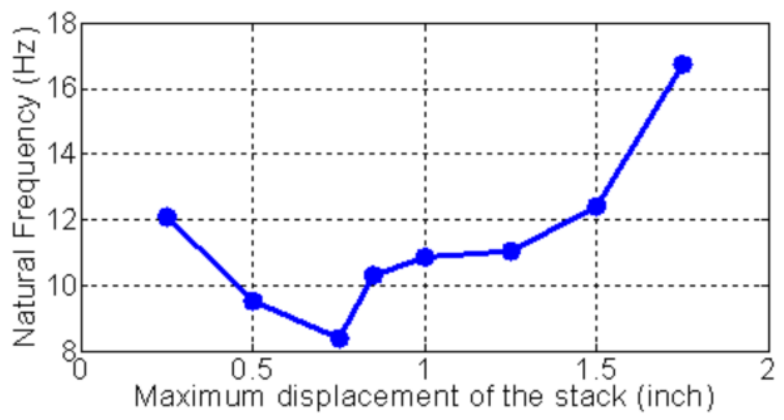

Fig. 14 Natural frequency versus maximum displacement of the magnet stack during the vibration of the system

Also, it should be noted that there is a trade-off for reducing the natural frequency since decreasing the displacement of the magnets stack will result in a decrease in the power output of the system (based on the Faraday's law). Therefore, there is an optimum value for each specific design in terms of minimum natural frequency and the efficient power output.

\section{RMS Prototyping}

In this section, the RMS prototyping is presented in detail. First, the assembling procedure of the moving magnets stack is described, which consists of forming three magnets and two pole pieces into a final product.

\subsection{Magnets stack assembling}

Since the RMS has three magnets facing each other from the same poles, the assembly procedure of the stack might be a little problematic due to the repelling force of the magnets. Pole pieces are considered to have $0.5 \mathrm{in}$. $(12.7 \mathrm{~mm})$ thickness and in order to help putting magnets close together, a rod with the diameter of $0.25 \mathrm{in}$. $(6.35 \mathrm{~mm}$, the same as the inner diameter of magnets) is threaded on its outside. Also, pole pieces are threaded on the inside to use the screw/nut mechanism to assemble the magnet stack easier and with less force.

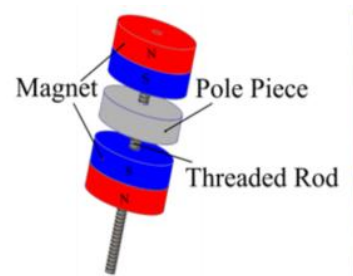

(a)

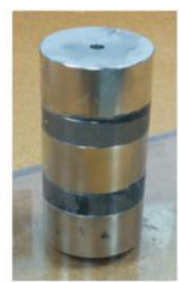

(b)

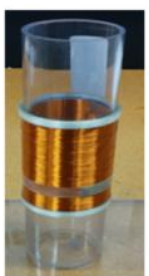

(c)

Fig. 15 (a) Assembly procedure of the magnets stack, (b) assembled magnet stack, (c) coil winded tube

In this work, 1018 steel for pole piece and 305 stainless steel for the rod were used. The 305 stainless steel is used to fabricate the screw mechanism due to the fact that it is almost non-magnetic. JB Weld glue is used to keep the magnets strongly tight together under harsh vibrations.

The next step is to wind the coil around a 10 inch long polycarbonate tube (see Table 6 and 7 for parametric details). A total 10 layers of an AWG 35 wire is wound using a computerized coil winding machine. Fig. 15 (c) shows the tube with the wire. Fig. 16 shows the finished prototype ready for experimental testing. 


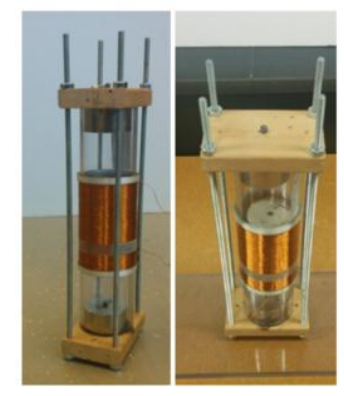

Fig. 16 Energy harvester prototype

Table 6 Characteristics of the Tube

\begin{tabular}{cccc}
\hline $\begin{array}{c}\text { Outer Diameter } \\
\text { (in) }\end{array}$ & $\begin{array}{c}\text { Inner Diameter } \\
\text { (in) }\end{array}$ & Material & $\begin{array}{c}\text { Length } \\
\text { (in) }\end{array}$ \\
$2.5(63.5 \mathrm{~mm})$ & $2.25(57.15 \mathrm{~mm})$ & Polycarbonate & $10(254 \mathrm{~mm})$ \\
\hline
\end{tabular}

Table 7 Characteristics of the coil

\begin{tabular}{ccc}
\hline Length (in) & $\begin{array}{c}\text { Number of } \\
\text { layers }\end{array}$ & Wire type \\
$4(101.6 \mathrm{~mm})$ & 10 & AWG 35 \\
\hline
\end{tabular}

\section{Experiments}

Experiments are conducted to verify the energy harvesting performance of the RMS prototype. As shown in Figure 17, the RMS is mounted on a shaker and an oscilloscope is used to measure the emf voltage output under the applied base sinusoidal excitation force.

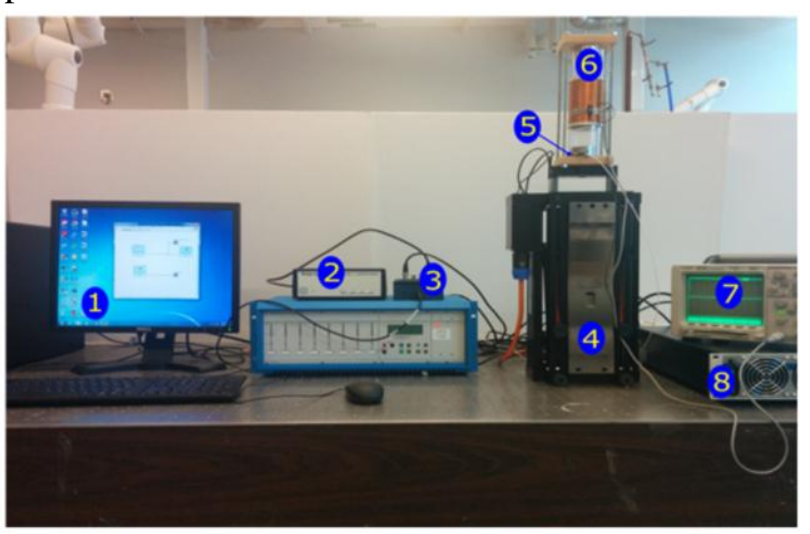

Fig. 17 Experimental setup: (1) Computer with LabView software, (2) PCB sensor signal conditioner, (3) Data acquisition, (4) Shaker, (5) PCB accelerometer, (6) Harvester, (7) Oscilloscope, (8) Power supply

The excitation signal is measured by using an accelerometer and it is a sine signal with $3.4 \mathrm{~g}$ amplitude and $9 \mathrm{~Hz}$ frequency. Five seconds of the excitation force signal are shown in Fig. 18. 


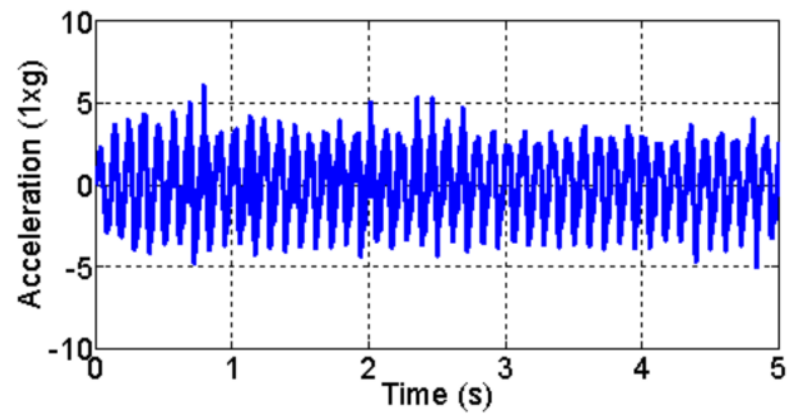

Fig. 18 Excitation signal $\left(\mathrm{g}=9.81 \mathrm{~m} / \mathrm{s}^{2}\right)$

The maximum amplitude of the signal is around 3.4g, where $\mathrm{g}$ is the gravitational force. Applying the above-mentioned excitation force to the base of the harvester using the shaker results in an emf voltage output, measured by an oscilloscope. The voltage output is shown in Fig. 19.

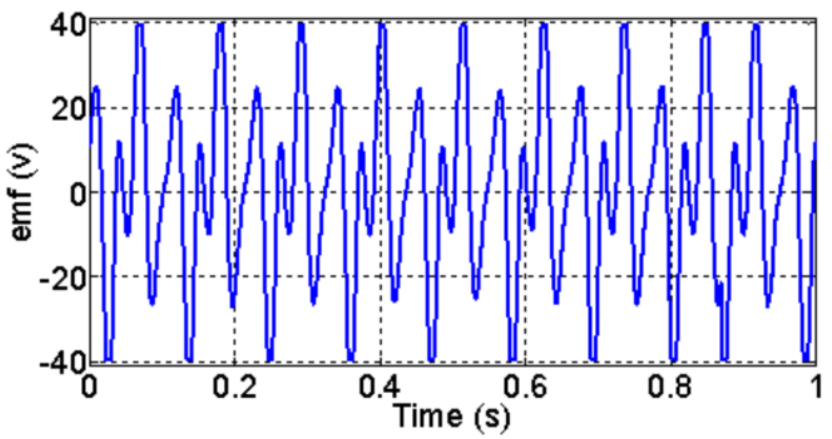

Fig. 19 Voltage output of the device under the sinusoidal vibration force with $9 \mathrm{~Hz}$ frequency

As it can be seen from Fig. 19, the maximum voltage reaches 41.18 volts in each cycle. In the next step, the effect of excitation frequency on the output voltage is discussed to see how the emf voltage changes by changing the excitation frequency. To do this, the output voltage is measured for each frequency ranging from $6 \mathrm{~Hz}$ to $12 \mathrm{~Hz}$. Results are shown in Fig. 20.

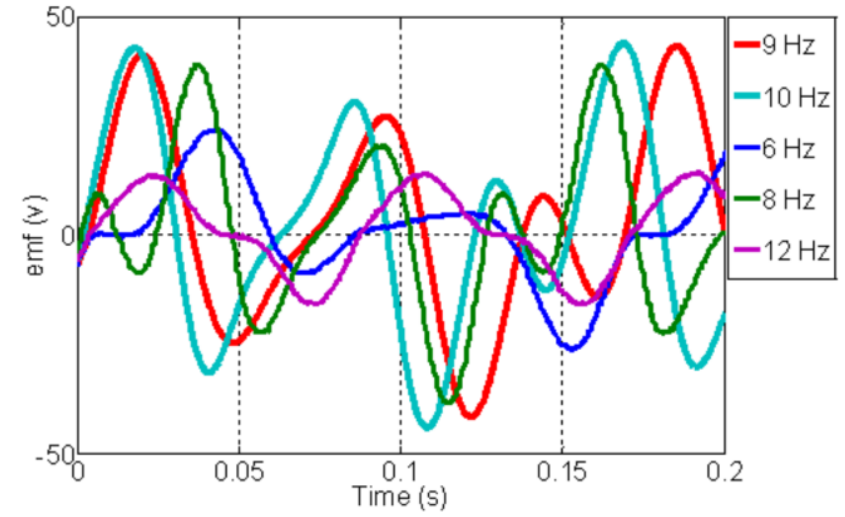

Fig. 20 Emf voltage of the RMS device under the sinusoidal excitation force for different excitation frequency for 0.2 second

The maximum emf voltage is found to be under excitation of around $9 \sim 10 \mathrm{~Hz}$ and the voltage output decreases while the excitation frequency moves away from this range. The maximum value of emf voltage for each excitation frequency is listed in Table 8. 
Table 8 Maximum emf voltage for different excitation frequencies and 3.4g amplitude

\begin{tabular}{cccccc}
\hline $\begin{array}{c}\text { Frequency } \\
(\mathrm{Hz})\end{array}$ & 6 & 8 & 9 & 10 & 12 \\
Voltage (v) & 24.1 & 39.1 & 41.18 & 42.86 & 13.8 \\
\hline
\end{tabular}

Finally, in order to investigate the influence of the amplitude of the sinusoidal vibration force on emf voltage output, different tests are performed by applying excitation forces with different amplitudes. The amplitude varies from $1.22 \mathrm{~g}$ up to $3.41 \mathrm{~g}$ while the frequency is kept at $9 \mathrm{~Hz}$. Results are shown in Fig. 21.

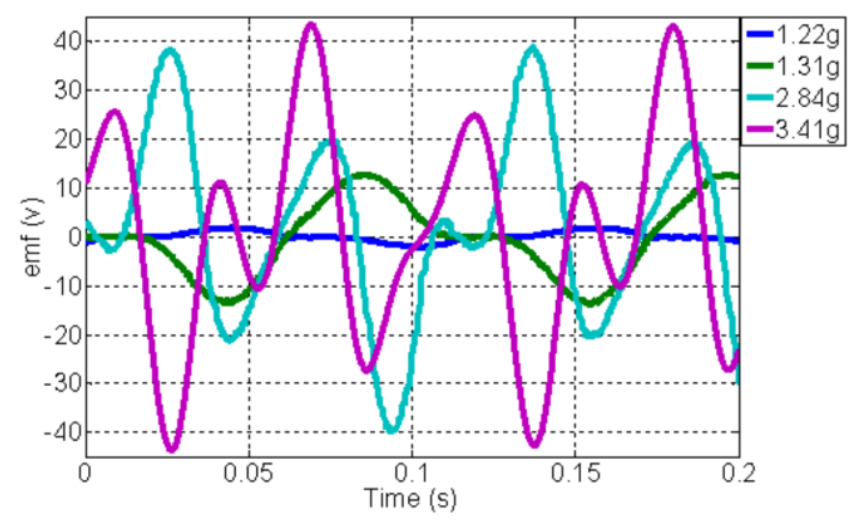

Figure 21 Emf voltage of the device under the sinusoidal excitation force with different amplitudes and

$9 \mathrm{~Hz}$ frequency

As expected, increasing the amplitude of the excitation force leads to the increment of emf voltage output. It can be seen that using a sinusoidal excitation force with $9 \mathrm{~Hz}$ frequency and a $1.22 \mathrm{~g}$ amplitude provides us with an emf voltage output of around 1.94 volts. The maximum value of an emf voltage for excitation forces with different amplitudes are listed in Table 9.

Table 9 Maximum emf voltage for excitation forces with different amplitudes

\begin{tabular}{ccccc} 
Amplitude & $1.22 \mathrm{~g}$ & $1.31 \mathrm{~g}$ & $2.84 \mathrm{~g}$ & $3.41 \mathrm{~g}$ \\
Voltage (v) & 1.94 & 12.8 & 38.4 & 43.4 \\
\hline
\end{tabular}

As one can notice, the shape of the emf voltage signal is different from the one we found using the numerical simulations. The main reason could be the unpredictable damping characteristic of the system. Since we are not using any lubricating procedure, there is coulomb friction between the moving stack and the tube as opposed to the viscous damping model that we used to model and simulate the system. Such a change in damping phenomenon leads to a different vibration behaviour of the system and consequently provides an emf voltage output with a different shape. Also, there are other reasons for deviation of the numerical simulation of the output emf from the experimental results, including the probable slight difference between magnetic field values obtained from the FEM analysis and real values and also coil position that might not be exactly as it is in the experiment during the vibration.

The experimental procedure used to verify the numerical modelling was explained and employed to provide some insight into the possibility of some practical applications for this device. Granted manufacturing very large scale prototypes might have difficulties and some modifications in the design should be considered, the procedure can be used to manufacture and assemble mid-size harvesters, i.e. 3 to 4 times larger than the current device. Larger prototypes will have lower natural frequencies due to 
their larger mass and the stack of magnets will move much slower with a lower frequency which is way closer to the typical high-density-frequencies of the ocean waves. It should be noted that the application of this design is not limited to ocean wave evergy harvesters and dpending on the size and tunned frequencies it can be used to harvest environmental vibrations in different situations such as bridges, railroads, bikes, transportation vehicles, and etc.

Finally, the experiments conducted in this paper were only for the purpose of explaining the manufacturing procedure and the method which can be used to test and measure the output power. The same process can be used to perform more experiments to study the effect of distance between magnets and also tunning method discussed in section 3.6. As it can be seen in Fig.20 and Fig.21, the change in frequency and the amplitude of the force signal applied to RMS resulted in changes in frequencies and amplitude of the output signal. This tunning procedure and how it can be implemented to design a harvester with a frequency close ot the desired optimum frequency is a topic of the next study by the authors and will be investigated in depth and with details.

\section{Conclusion and remarks}

This work extensively studied a nonlinear energy harvester RMS which works based on a magnetic levitation mechanism with repulsive magnetic force. The design is beneficial in terms of minimum number of moving mechanical parts as well as a stronger magnetic field, leading to a high voltage output. The system was numerically modelled to evaluate the magnetic field strength for different magnet arrays and configurations. Also, nonlinear vibration response and the resulted voltage and power output were estimated using numerical methods for the RMS. The device has a tunable resonance frequency, which is a function of the maximum displacement of the magnets stack during the course of vibration. This change of the resonance has an optimum point that we can reach by changing the maximum displacement for each specific harvester design. Finally, to experimentally evaluate the numerical procedure, a prototype of the RMS was manufactured and the base-excitation vibration tests was performed. Results show that by using an excitation force with the maximum amplitude of circa $3.4 \mathrm{~g}$ and the frequency of $9 \mathrm{~Hz}$, the maximum emf voltage of 42 volts can be achieved. The effects of the excitation frequency and the force amplitude were also studied. The parametric study was performed to provide some insights into the effects of the excitation force characteristics on the RMS output. The prototype, studied and fabricated in this work, is an example of how this mechanism can be used for ocean wave energy harvesting. However, to capture the wave energy using this mechanism, it is essential to design a system with a different size and a very low frequency resonance. Moreover, to accurately simulate the vibrations of the system, either coulomb damping model should be used instead of the viscous damping model or a lubrication method should be implemented which also improves the emf output voltage for low amplitude vibrations. This deign procedure can be directly implemented for an oscillating water column (OWC) ocean wave energy harvester, however, it might bring difficulties for prototypes with larger sizes due to the limitations in the size of the magnets available and the installation of the magnets. Furthermore, with some modifications, the repulsive force between magnets can be used in smaller sections of a very large OWC as additional features to optimize the output energy. The possible techniques to scale up the design for OWC and how it might also be practical to add it as a feature to the current OWCs to increase their efficiency will be part of the topic and discussions in the next work of these authors. 


\section{AKNOWLEDMENT}

The authors would like to thank the support of DOE ARPA-E under grant DOE-AR0000531. The authors gratefully acknowledge the comments and advice of Dr. Reed E. Phillips from Energystics Ltd regarding the design of the RMS. The authors like to thank Banu Chandan Sajja for the help with experimental testing.

\section{REFERENCES}

[1] R.,Waters, Energy from ocean waves: Full scale experimental verification of a wave energy converter, PhD Thesis, Uppsala University, 2008.

[2] J.,Falnes, and J., Løvseth, "Ocean wave energy," Energy Policy, 19 (1991), pp. 768-775.

[3] T.W., Thorpe, A brief review of wave energy, Harwell Laboratory, Energy Technology Support Unit Report Number R-120, London, UK, 1999.

[4] J., Brooke, Wave Energy Conversion, Elsevier, Amsterdam, 2003.

[5] D., Ross, Power from the Waves, Oxford University Press, USA, 1995.

[6] A., Clément, P., McCullen, A., Falcão, A., Fiorentino, F., Gardner, K., Hammarlund, G., Lemonis, T., Lewis, K., Nielsen, and S., Petroncini, Wave energy in Europe: current status and perspectives, Renewable and Sustainable Energy Reviews, 6 (2002), pp. 405-431.

[7] T., Heath, A review of oscillating water columns, Philosophical Transactions of the Royal Society A: Mathematical, Physical and Engineering Sciences, 370 (2012), pp. 235-245.

[8] J., Falnes, Radiation impedance matrix and optimum power absorption for interacting oscillators in surface waves, Applied Ocean Research, 2 (1980), pp. 75-80.

[9] J., Falnes, A review of wave-energy extraction, Marine Structures, 20 (2007), pp. 185-201.

[10] K., Rhinefranka, E.B., Agamloha, A., von Jouannea, A.K., Wallacea, J. Prudella, K., Kimblea, J., Aillsa, E., Schmidta, P., Chanb, B., Sweenyb, and A., Schachera, Novel ocean energy permanent magnet linear generator buoy, Renewable Energy, 31 (2006), pp. 1279-1298

[11] K., Thorburn, and M., Leijon, Ocean Engineering, 34 (2007), pp. 908-916

[12] M. J., Brennana, I., Kovacicb, A. Carrellaa, and T.P., Waters, Journal of Sound and Vibration, 318 (2008), pp. 1250-1261

[13] N.M., Kimoulakis, A.G., Kladas, and J.A., Tegopoulos, Power Generation Optimization From Sea Waves by Using a Permanent Magnet Linear Generator Drive, IEEE TRANSACTIONS ON MAGNETICS, 44 (2008), pp. 1530-1533

[14] D., Elwood, S.C., Yim, E., Amon, A., von Jouanne, and T.K.A., Brekken, Experimental Force Characterization and Numerical Modeling of a Taut-Moored Dual-Body Wave Energy Conversion System, Journal of Offshore Mechanics and Arctic Engineering, 132 (2010)

[15] J., Prudell, M., Stoddard, E., Amon, T.K.A. Brekken, and A., von Jouanne, A Permanent-Magnet Tubular Linear Generator for Ocean Wave Energy Conversion, IEEE TRANSACTIONS ON INDUSTRY APPLICATIONS, 46 (2010), pp. 2392-2400

[16] D., Su, K., Nakano, R., Zheng, and M.P., Cartmell, On electrical optimisation using a Duffing-type vibrational energy harvester, Proceedings of the Institution of Mechanical Engineers, Part C: Journal of Mechanical Engineering Science, 229 (2014), pp. 3308-3319.

[17] B.,Mann, and N., Sims, N., Energy harvesting from the nonlinear oscillations of magnetic levitation, Journal of Sound and Vibration, 319 (2009), pp. 515-530. 